\title{
In-situ growth studies of sputtered YBCO thin films by spectroscopic ellipsometry
}

\author{
M.E. Bijlsma*, D.H.A. Blank, H. Wormeester, A. van Silfhout, H. Rogalla \\ Faculty of Applied Physics, University of Twente, P.O. Box 217, 7500 AE, Enschede The Netherlands
}

\begin{abstract}
Using spectroscopic ellipsometry we studied in-situ the growth of off-axis sputtered $\mathrm{YBa}_{2} \mathrm{Cu}_{3} \mathrm{O}_{6+x}$ thin films on (001) SrTiO, as a function of the deposition parameters. Especially in the very first growth stage $(<5 \mathrm{~nm})$ we observed that the optical properties of the grown layer differs from the "bulk" optical pronerties of YBCO and strongly depends on, both, the deposition temperature and the oxygen partial pressure. Both properties are well established as influencing the superconducting properties of thin YBCO films. YBCO thin film growth under optimal deposition conditions $\left(T_{c} \sim 90 \mathrm{~K} ; j_{c}>10^{6} \mathrm{~A} \mathrm{~cm}^{-2} @ 77 \mathrm{~K}\right)$ is smooth and homogeneous, except for the first unit cell layer (initial stage regime). The smoothness of the response is indicative for a step-mode like growth mechanism. In contrast. the initial stage regime is governed by a 2D nucleation mechanism. This behaviour changes when the deposition temperature is lowered. Due to increased disorder, the initial stage regime is extended to larger thicknesses and a true 2D growth mode is no longer apparent. Similar behaviour is observed with increasing oxygen partial pressure, where the optical response is shifted from a step-flow mode like mechanism to an island-growth mode.
\end{abstract}

Keywords: Sputtered YBCO thin-films: Spectroscopic ellipsometry

\section{Introduction}

The hetero-epitaxial growth of YBCO on STO in the initial growth regime has been a rather controversial issue for several years already. On the one hand, observed RHEED (reflection high energy electron diffraction) intensity oscillations during the growth process points to a 2D layer-by-layer growth mode $[1-3]$. On the other hand, STM/AFM (scanning tunneling microscope)/(atomic force microscope) studies on very thin films $[4,5]$ and the RHEED study of Chandrasekhar et al. [6] rather suggest an island-growth mode.

In this paper, we report on in-situ ellipsometry studies in the initial growth regime of the $\mathrm{YBa}_{2} \mathrm{Cu}_{3} \mathrm{O}_{6+x}$ sputter deposition process. Ellipsometry is non-destructive and can be used in any transparent medium. The major disadvantage of ellipsometry is the indirectness of the technique. The measured effective optical response of the structure under investigation can only be related to actual material properties by a model calculation. Nevertheless, the realtime application of ellipsometry has proved to be a

\footnotetext{
*Corresponding author.
}

successful growth analyzing instrument with sub-nanome= ter depth resolution. [7].

\section{Experimental}

$\mathrm{YBa}_{2} \mathrm{Cu}_{3} \mathrm{O}_{6+x}$ thin films are deposited in an off-axis $\mathrm{RF}$ (radio frequency) magnetron sputter system. Details can be found elsewhere [8]. Under optimized deposition con= ditions, $T_{\text {Jep }}=770^{\circ} \mathrm{C}, P_{\text {tot }}=20 \mathrm{~Pa}, \mathrm{Ar}: \mathrm{O}_{2}$ ratio $=3.4, P_{\mathrm{rr}}$ $90 \mathrm{~W}$ and a subsequent high pressure oxygen anneal step. high quality superconducting thin films can be obtained with $T_{c, 0}=90 \mathrm{~K}$ and $j_{r}>1 \cdot 10^{6} \mathrm{~A} \mathrm{~cm}^{-2}$ at $77 \mathrm{~K}$. Films are then fully c-axis textured.

Ellipsometric measurements were performed using a $P_{\text {rot }}$ SA scanning monochromator configuration attached on the deposition chamber. Measurements were performed at a 69.1 degrees angle of incidence. Real-time data collection was performed at a single photon energy with an averaged data cycle time of $10 \mathrm{~s}$. Ellipsometric angles $\psi_{\mathrm{m}}$ and $\Delta_{\mathrm{m}}$ could be obtained with a measurement precision better than $0.1^{\circ}$ for $\Delta$ and $0.05^{\circ}$ for $\psi$. Optical simulations are performed in a full $4 \times 4$ matrix formalism framework [9] in order to include the optical anisotropy of 
$\mathrm{YBa}_{2} \mathrm{Cu}_{3} \mathrm{O}_{6+\ldots}$. Non homogeneous growth was simulated using the self-consistent Bruggeman effective medium approximation. Layer nucleation was simulated using hemispherical nuclei on a square grid increasing in size. Results of model calculations were iterated towards the experimental values using a standard linear regression analysis.

\section{Results and discussion}

\subsection{Deposition temperature effects}

The initial growth behaviour at different deposition temperatures maintaining all other deposition parameters fixed is depicted in Fig. 1. The traces can be oivided into four regions with thickness increments, which are full c-axis unit cell multiples. The different starting points in the $\Delta-\psi$ plane for the various samples are a result of the use of different substrates with slightly varying optical properties. Considering the sample grown at $800^{\circ} \mathrm{C}$ (sam. ple A), we observe a smooth growth behaviour already at a thickness in the order of $1 \mathrm{~nm}$. The only anomalous behaviour is restricted to region I. Lowering the temperature towards $740^{\circ} \mathrm{C}$ (sample B) increases the apparent anomalous changes towards region II. The same is true for the $720^{\circ} \mathrm{C}$ sample (sample C), A further decrease in temperature (samples D and E) introduces a faint slowly changing additional structure in the optical response in region III. The growth behaviour in region IV is essentially the same for all samples and shows a near linear change of the optical response with increasing thickness, indicative of a smooth and homogeneous film growih process.

Simulating the initial growth stage at a temperature of $800^{\circ} \mathrm{C}$ with a simple step-llow model (Fig. 2), excellent agreement in the thickness region $>1 \mathrm{~nm}$ is achieved. The lirst unit cell layer formation is simulated by a pure lateral development of unit cell high material. The first layer has to be filled completely, before nucleation of a second and

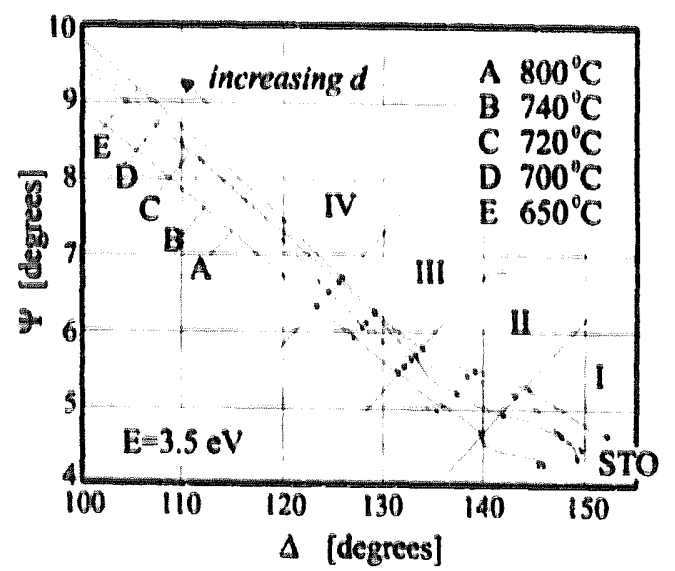

Fig. 1. $\mathrm{YBa}_{2} \mathrm{Cu}_{8} \mathrm{O}_{n,}$, initial growth optical response on STO as a function of deposition temperature. The photon energy is $E=3.5 \mathrm{eV}$.

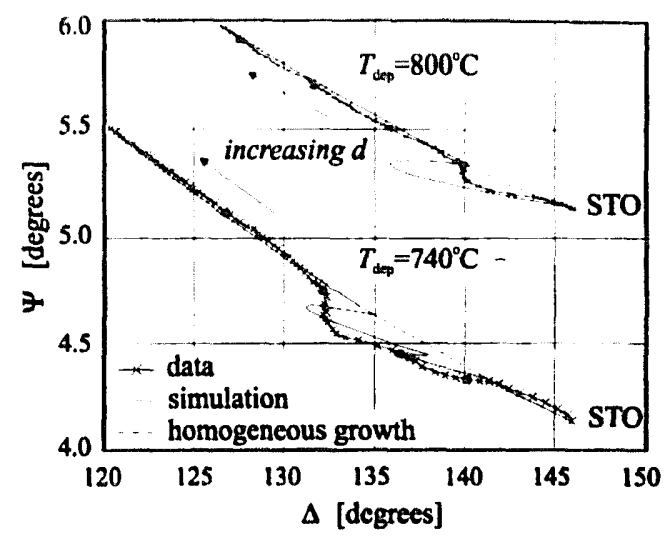

Fig. 2. $\mathrm{YBa}, \mathrm{Cu}_{1} \mathrm{O}_{0} .$. initial growth response on $\mathrm{STO}$ at $T_{\mathrm{tep}}=800^{\circ} \mathrm{C}$ and $T_{\text {dep }}=740^{\circ} \mathrm{C}$ : the upper curve is shifted one degree upward for clarity. Black dots indieate I mn thickness increments. The photon energy is $E=3.5 \mathrm{eV}$.

subsequent layers occurs. Hence, coalescence is fast, which is supported by resistivity measurements $[4,10]$.

The full cusped structure amplitude predicted by the ideal case simulation of the formation of the first layer is not fully reached by the data points, which is understandable considering that the data points represent an optical response averaged over a large film area $\left(\approx 0.2 \mathrm{~cm}^{2}\right)$. The true 2D growth mode in the first growth stage is in clear accordance with the RHEED studies of Karl and Stritzker |2| and Chern et al. 1111. Also the subsequent step.flow growth is supported by the observed strong RHEED oscillation damping (also within two unit cell layers), when measurements are performed on vicinal substrates |2|. In our ease, for sumples grown at $800^{\circ} \mathrm{C}$. we deduce diffusion lengths in the order of $50 \mathrm{~nm}$ as determined by STM from the typieal size of terraces. This diffusion length is substantially larger than the substrate lerrace length, which was estimated to be in the order of $20 \mathrm{~nm}$. Reduction of the temperature decreases the diffusion length considerably and will prevent or reduce step-flow growth and impose island nucleation on the terraces. The increased nucleation probability will make the optical response more 3D like. as more different unit cell layers will be incorporated in the growth process. Fan and Somekh $|4|$ reported in an AFM study, of nucleation of flat islands of one unit cell high. which develop laterally as the film thickness increases to $2-3 \mathrm{~nm}$ and coalescence at about 3-4 nm. We observed similar behaviour (Fig. 2) at $r_{\mathrm{den}}=740^{\circ} \mathrm{C}$. Note the still present unit cell thick modulation in the response. The rather smooth hehaviour up 10 I $\mathrm{nm}$ thickness suggests an extension of a film region. whose origin can also be seen in the $800^{\circ} \mathrm{C}$ case (the region with $\Delta>145^{\circ}$ ), where this region is much less apparent due to its decreased thickness. Probably, this layer represents a thin interface accommodating layer with a high density of phases other than the 123-phase. On top of this accommodating layer, a double unit cell thick structure must be used to simulate the optical growth response. The nearly smooth behaviour near 
$2 \mathrm{~nm}$ thickness indicates a quick nucleation of a subsequent unit cell layer before the bottom layer is comfleted. Also in this case, the optical response recovers almost totally to the homogeneous growth response behaviour and after $3 \mathrm{~nm}$ film thickness, the smooth response is again dominant. Upon a further reduce in temperature to $T_{\text {dep }}=720^{\circ} \mathrm{C}$ and lower, the initial stage optical response smoothens and extends towards larger thicknesses (up to 7 $\mathrm{nm}$ ) beiore the linear response region is reached. The smoother behaviour can be explained by the possibility of a more island like response, where several ( $\sim 3-5$ layers) determine simultaneously the observed optical response, or by a larger disorder at the interface, which smoothens (or better obscures) the optical response behaviour. Accordingly, simulation attempts will have a limited physical meaning in these cases.

\subsection{Oxygen pressure effects}

The initial growth behaviour of YBCO on STO is not only strongly dependent on the depusition temperature, but is also influenced by the oxygen partial background pressure during growth. In Fig. 3, the ellipsometric response in the initial growth regime is given for four different thin films grown with different oxygen partial pressures. The deposition temperature is constant at $T_{\text {dep }}=$ $770^{\circ} \mathrm{C}$. Trace B can be directly compared to the similar optical response at a iemperature of $800^{\circ} \mathrm{C}$ of Fig. 2. Also now, a homogeneous, smooth growth response is obtained except in the first $1 \mathrm{~nm}$ deposition stage. Although a decrease in the oxygen partial pressure (ratio $\mathrm{Ar}_{2} \mathrm{O}_{2}=1: 1$ ) deteriorates the superconducting properties significantly. no influence on the optical response is observed in the initial growth regime (trace $A$ ). The reduction of the superconducting properties is connected to a $c$-axis enlargement, caused by the introduction of small structural defects (oxygen disorder). Such defects are likely to influence predominantly the "bulk" behaviour. Only when

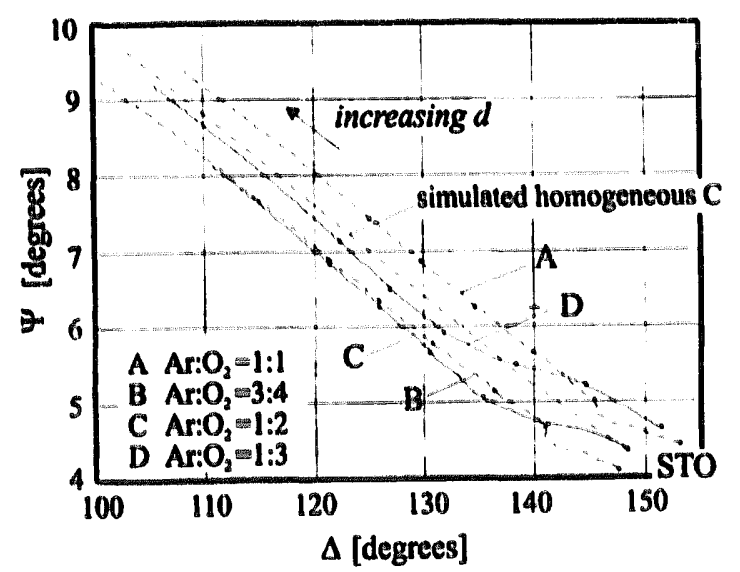

Fig. 3. $\mathrm{YBu}_{2} \mathrm{Cu}_{3} \mathrm{O}_{0+x}$ initial growth optical response on $\mathrm{STO}$ as a function of oxygen partial pressure at $r_{\mathrm{Jep}}=770^{\circ} \mathrm{C}$. Black dots indicate I $\mathrm{nm}$ thickness increments. The photon energy is $E=3.5 \mathrm{eV}$. the oxygen pressure is substantially further reduced, can influence be expected in the initial growth response. In contrast, the initial growth response is strongly influenced by a high oxygen partial pressure (trace $C$ and $D$ ). The response smoothens and extends over a larger thickness $(\approx 5 \mathrm{~nm})$ compared to the $3: 4$ ratio. In both cases, the response is similar and resembles an island-growth mode optical response, as can be expected from the reduction of the atomic mobility on the surface with increasing oxygen amount. The increase of the oxygen pressure, with respect to the 3:4 ratio, does not significantly change the superconducting properties of the thin films. This behaviour is an indication, that at higher oxygen pressures, the oxygen is rather excessive and is not necessary in the formation process of the 123-phase. Nevertheless, excess oxygen does play a role in the formation process of excess oxidic phases. Such secondary phases man appear on the surface or may be incorporated in the film. From our measurements, we cannot deduce whether only the 123-phase nucleates on the surface or whether other phases may contribute in the nucleation and coalescence process. The gradual response in the first nanometer thickness regime of trace $C$ and $D$ can only point to an increased disorder in the first stages of growth as compared to trace B. Interestingly, the traces $C$ and $D$ never approach the homogeneous growth response trace (dotted curve) at larger film thick. nesses. This behaviour is in contrast with the optical response at reduced deposition temperatures, which does show this approach to the homogeneous growth situation. The effect can have two reasons. Firstly, a 123 phase island-growth mechanism is induced by the excess oxygen. Here, typical island sizes are likely to decrease with increasing pressure. In this case, several unit cell layers simultancously are involved in the optical response and a continuous deviation from the homogeneous growth opti: cal response can be expected. The other possibility is the presence of disorder inside the film as a result of secondary phases, which are easily formed by the excess oxygen. As a consequence, the effective dielectric constant of the film is influenced and will differ from the homogeneous growth dielectric constant value.

\section{Conclusions}

In-situ ellipsometric measurements in the initial growth regime reveal that YBCO thin film growth under optimal deposition is governed by a step-flow growth mode except for the first unit cell layer. We attribute deviations in the ellipsometric response during the first unit cell deposition to a 2D nucleation mechanism. Upon lowering of the deposition temperature, the optical response changes. Comparison with simulations indicates a cross-over to a 3D layer nucleation mechanism accompanied by an increased disorder at the surface.

Similar behaviour can be observed, when the oxygen 
partial pressure is increased. The initial growth regime then changes from a one unit cell high 2D growth mode regime to a much thicker optically smooth region. The behaviour can both be explained by a cross-over to an island-growth mode for higher oxygen pressures and by the increasing appearance of oxidic, secondary phases, which introduce disorder in the early stages of 123-phase thin film growth.

\section{References}

III T. Terashima, Y. Bardo, K. Jjima, K. Yamamoto, K. Hirata. K. Hayashi, K. Kamigaki and H. Teranchi. Phys. Rev. Lett. 65 (1990) 2684.
(2) H. Karl and B. Stritzker, Phys. Rev. Lett. 69 (1992) 2939.

[3] T. Frey, C.C. Chi, C.C. Tsuei, T. Shaw and F. Boszo. Phys. Rev.. B49 (1994) 3483.

[4] S.C. Fan and R.E. Somekh, presented at Eucas'95.

[5] D.G. Schiom, D. Anselmetti, J.G. Bednorz, R.F. Broom, A. Catana, T. Frey, Ch. Gerber, H.J. Guntherodi, H.P. Lang and J. Mannhart, $Z$. Phys., B86 (1992) 163.

[6] N. Chandrasekhar, V.S. Achutharaman, V. Agrawal and A.M. Goldman, Phys. Rev.. B46 (1992) 8565.

[7] J.B. Theeten, Surf. Sci., 96 (1980) 275.

[8] M.A.J. Verhoeven, Ph.D. Thesis, University of Twente, The Netherlands, 1996.

[9] P. Yeh. J. Opt. Soc. Am., 69 (1979) 742.

[10] X.X. Xi, J. Geerk, G. Linker, Q. Li and O. Meyer, Appl. Phys. Lett., 54 (1989) 2367.

(II) M.Y. Chern, A. Gupta and B.W. Hussey, Appl. Phys. Lett. 60 (1992) 3045. 